PROCEEDINGS OF THE

AMERICAN MATHEMATICAL SOCIETY

Volume 137, Number 5, May 2009, Pages 1561-1567

S 0002-9939(08)09742-6

Article electronically published on November 13, 2008

\title{
INDUCED QUASI-ACTIONS: A REMARK
}

\author{
BRUCE KLEINER AND BERNHARD LEEB
}

(Communicated by Alexander N. Dranishnikov)

\begin{abstract}
We observe that the notion of an induced representation has an analog for quasi-actions and give some applications.
\end{abstract}

\section{INTRODUCTION}

In this paper we observe that the notion of an induced representation has an analog for quasi-actions and give some applications.

We will use the definitions and notation from KL01.

1.1. Induced quasi-actions and their properties. Let $G$ be a group and $\left\{X_{i}\right\}_{i \in I}$ be a finite collection of unbounded metric spaces.

Definition 1.1. A quasi-action $G \stackrel{\rho}{\curvearrowright} \prod_{i} X_{i}$ preserves the product structure if each $g \in G$ acts by a product of quasi-isometries, up to a uniformly bounded error. Note that we allow the quasi-isometries $\rho(g)$ to permute the factors; i.e. $\rho(g)$ is uniformly close to a map of the form $\left(x_{i}\right) \mapsto\left(\phi_{\sigma^{-1}(i)}\left(x_{\sigma^{-1}(i)}\right)\right)$ with a permutation $\sigma$ of $I$ and quasi-isometries $\phi_{i}: X_{i} \mapsto X_{\sigma(i)}$.

Associated to every quasi-action $G \stackrel{\rho}{\curvearrowright} \prod_{i} X_{i}$ preserving product structure is the action $G \stackrel{\rho_{I}}{\curvearrowright} I$ corresponding to the induced permutation of the factors; this is well-defined because the $X_{i}$ 's are unbounded metric spaces. For each $i \in I$, the stabilizer $G_{i}$ of $i$ with respect to $\rho_{I}$ has a quasi-action $G_{i} \curvearrowright X_{i}$ by restriction of $\rho$. It is well-defined up to equivalence in the sense of [KL01, Definition 2.3].

If the permutation action $\rho_{I}$ is transitive, all factors $X_{i}$ are quasi-isometric to each other, and the restricted quasi-actions $G_{i} \curvearrowright X_{i}$ are quasi-conjugate (when identifying different stabilizers $G_{i}$ by inner automorphisms of $G$ ). The main result of this note is that in this case any of the quasi-actions $G_{i} \curvearrowright X_{i}$ determines $\rho$ up to quasi-conjugacy, and moreover any quasi-conjugacy class may arise as a restricted action.

Theorem 1.2. Let $G$ be a group, $H$ be a finite index subgroup, and $H \stackrel{\alpha}{\curvearrowright} X$ be a quasi-action of $H$ on an unbounded metric space $X$. Then there exists a quasiaction $G \stackrel{\beta}{\curvearrowright} \prod_{i \in G / H} X_{i}$ preserving product structure, where

(1) Each factor $X_{i}$ is quasi-isometric to $X$.

Received by the editors February 25, 2008, and, in revised form, June 23, 2008.

2000 Mathematics Subject Classification. Primary 20F65.

The first author was partially supported by NSF Grant DMS 0701515.

(C)2008 American Mathematical Society 
(2) The associated action $G \stackrel{\beta_{G / H}}{\curvearrowright} G / H$ is the natural action by left multiplication.

(3) The restriction of $\beta$ to a quasi-action of $H$ on $X_{H}$ is quasi-conjugate to $H \stackrel{\alpha}{\curvearrowright} X$.

Furthermore, there is a unique such quasi-action $\beta$ preserving the product structure, up to quasi-conjugacy by a product quasi-isometry. Finally, if $\alpha$ is an isometric action, then the $X_{i}$ may be taken isometric to $X$ and $\beta$ may be taken to be an isometric action.

Definition 1.3. Let $G, H$ and $H \curvearrowright X$ be as in Theorem 1.2. The quasi-action $\beta$ is called the quasi-action induced by $H \curvearrowright X$.

As a byproduct of the main construction, we get the following:

Corollary 1.1. If $G \stackrel{\rho}{\curvearrowright} X$ is an $(L, A)$-quasi-action on an arbitrary metric space $X$, then $\rho$ is $(L, 3 A)$-quasi-conjugate to a canonically defined isometric action $G \curvearrowright X^{\prime}$.

1.2. Applications. The implication of Theorem 1.2 is that in order to quasiconjugate a quasi-action on a product to an isometric action, it suffices to quasiconjugate the factor quasi-actions to isometric actions. We begin with a special case:

Theorem 1.4. Let $G \stackrel{\rho}{\curvearrowright} X$ be a cobounded quasi-action on $X=\prod_{i} X_{i}$, where each $X_{i}$ is either an irreducible symmetric space of noncompact type, or a thick irreducible Euclidean building of rank at least two, with cocompact Weyl group. Then $\rho$ is quasi-conjugate to an isometric action on $X$, after suitable rescaling of the metrics on the factors $X_{i}$.

Remarks. - Theorem 1.4 was stated incorrectly as Corollary 4.5 in [KL01]. The proof given there was valid only for quasi-actions which do not permute the factors.

- Rescaling of the factors is necessary, in general: if one takes the product of two copies of $\mathbb{H}^{2}$ where the factors are scaled to have different curvature, then a quasi-action which permutes the factors will not be quasi-conjugate to an isometric action.

We now consider a more general situation. Let $G \stackrel{\alpha}{\curvearrowright} \prod_{i \in I} X_{i}$ be a quasi-action, where each $X_{i}$ is one of the following four types of spaces:

(1) An irreducible symmetric space of noncompact type.

(2) A thick irreducible Euclidean building of rank/dimension $\geq 2$, with cocompact Weyl group.

(3) A bounded valence bushy tree in the sense of MSW03. We recall that a tree is bushy if each of its points lies within a uniformly bounded distance from a vertex having at least three unbounded complementary components.

(4) A quasi-isometrically rigid Gromov hyperbolic space which is of coarse type I in the sense of [KKL98, Sec. 3] (see the remarks below). A space is quasiisometrically rigid if every $(L, A)$-quasi-isometry is at distance at most $D=$ $D(L, A)$ from a unique isometry. Examples include rank 1 symmetric spaces other than hyperbolic and complex hyperbolic spaces Pan89, Fuchsian buildings BP00, Xie06, and fundamental groups of hyperbolic $n$-manifolds with nonempty totally geodesic boundary, $n \geq 3$ [KKLS, BKM]. 
By [KKL98, Theorem B], the quasi-action preserves the product structure, and hence we have an induced permutation action $G \curvearrowright I$. Let $J \subset I$ be the set of indices $i \in I$ such that $X_{i}$ is either a real hyperbolic space $\mathbb{H}^{k}$ for some $k \geq 4$, a complex hyperbolic space $\mathbb{C} \mathbb{H}^{l}$ for some $l \geq 2$, or a bounded valence bushy tree. Generalizing Theorem 1.4 we obtain:

Theorem 1.5. If the quasi-action $G_{j} \curvearrowright X_{j}$ is cobounded for each $j \in J$, then $\alpha$ is quasi-conjugate by a product quasi-isometry to an isometric action $G \stackrel{\alpha^{\prime}}{\curvearrowright} \prod_{i \in I} X_{i}^{\prime}$, where for every $i, X_{i}^{\prime}$ is quasi-isometric to $X_{i}$, and precisely one of the following holds:

(1) If $X_{i}$ is not a bounded valence bushy tree, then $X_{i}^{\prime}$ is isometric to $X_{i^{\prime}}$ for some $i^{\prime}$ in the $G$-orbit $G(i)$ of $i$.

(2) If $X_{i}$ is a bounded valence bushy tree, then so is $X_{i}^{\prime}$.

As in the previous corollary, it is necessary to permit $X_{i}^{\prime}$ to be nonisometric to $X_{i}$. Moreover, there may be factors $X_{i}$ and $X_{j}$ of type (4) lying in the same $G$-orbit, but which are not even homothetic, so it is not sufficient to allow rescaling of factors.

Proof. We first assume that the action $G \curvearrowright I$ is transitive. Pick $n \in I$. Then the quasi-action $G_{n} \curvearrowright X_{n}$ is quasi-conjugate to an isometric action $G_{n} \curvearrowright X_{n}^{\prime}$, where $X_{n}^{\prime}$ is isometric to $X_{n}$ unless $X_{n}$ is a bounded valence bushy tree, in which case $X_{n}^{\prime}$ is a bounded valence bushy tree but not necessarily isometric to $X_{n}$; this follows from:

- Hin90, Gab92, CJ94, Mar06 when $X_{n}$ is $\mathbb{H}^{2}$. Note that any quasi-action on $\mathbb{H}^{2}$ is quasi-conjugate to an isometric action.

- Sul81, Gro, Tuk86, Pan89, Cho96] when $X_{n}$ is a rank 1 symmetric space other than $\mathbb{H}^{2}$. Note that Sullivan's theorem implies that any quasi-action on $\mathbb{H}^{3}$ is quasi-conjugate to an isometric action. Also, the proof given in Chow's paper on the complex hyperbolic case covers arbitrary cobounded quasi-actions, even though it is only stated for discrete cobounded quasi-actions.

- KL97, Lee00 when $X_{n}$ is an irreducible symmetric space or Euclidean building of rank at least 2 .

- MSW03 when $X_{n}$ is a bounded valence bushy tree.

By Theorem 1.2, the associated induced quasi-action of $G$ is quasi-conjugate to the original quasi-action $G \curvearrowright \prod_{i \in I} X_{i}$ by a product quasi-isometry, and we are done.

In the general case, for each orbit $G(i) \subset I$ of the action $G \curvearrowright I$, we have a well-defined associated quasi-action $G \curvearrowright \prod_{j \in G(i)} X_{j}$ for which the theorem has already been established, and we obtain the desired isometric action $G \curvearrowright \prod_{i \in I} X_{i}^{\prime}$ by taking products.

Corollary 1.2. Let $\left\{X_{i}\right\}_{i \in I}$ be as above, and suppose $G$ is a finitely generated group quasi-isometric to the product $\prod_{i \in I} X_{i}$. Then $G$ admits a discrete, cocompact, isometric action on a product $\prod_{i \in I} X_{i}^{\prime}$, where for every $i, X_{i}^{\prime}$ is quasi-isometric to $X_{i}$, and precisely one of the following holds:

(1) $X_{i}$ is not a bounded valence bushy tree, and $X_{i}^{\prime}$ is isometric to $X_{i^{\prime}}$ for some $i^{\prime}$ in the $G$-orbit $G(i) \subset I$ of $i$.

(2) Both $X_{i}$ and $X_{i}^{\prime}$ are bounded valence bushy trees. 
Proof. Such a group $G$ admits a discrete, cobounded quasi-action on $\prod_{i \in I} X_{i}$. Theorem 1.5 furnishes the desired isometric action $G \curvearrowright \prod_{i} X_{i}^{\prime}$.

Remarks. - Corollary 1.2 refines earlier results Ahl02, KL01, MSW03.

- A proper Gromov hyperbolic space with cocompact isometry group is of coarse type I unless it is quasi-isometric to $\mathbb{R}$ [KKL98, Sec. 3].

- The classification of the four different types of spaces above is quasi-isometry invariant, with one exception: a space of type (1) will also be a space of type (4) iff it is a quasi-isometrically rigid rank 1 symmetric space (i.e. a quaternionic hyperbolic space or the Cayley hyperbolic plane [Pan89]). See Lemma 3.1.

- Two irreducible symmetric spaces are quasi-isometric iff they are isometric, up to rescaling [Mos73, Pan89, KL97. Two Euclidean buildings as in (2) above are quasi-isometric iff they are isometric up to rescaling KL97, Lee00.

\section{The CONSTRUCtion OF InDUCED QUASI-ACTIONS}

The construction of induced quasi-actions is a direct imitation of one of the standard constructions of induced representations. We now review this for the convenience of the reader.

Let $H$ be a subgroup of some group $G$, and suppose $\alpha: H \curvearrowright V$ is a linear representation. Then we have an action $H \curvearrowright G \times V$ where $(h,(g, v))=\left(g h^{-1}, h v\right)$. Let $E:=(G \times V) / H$ be the quotient. There is a natural projection map $\pi$ : $E \rightarrow G / H$ whose fibers are copies of $V$; this would be a vector bundle over the discrete space $G / H$ if $V$ were endowed with a topology. The action $G \curvearrowright G \times$ $V$ by left translation on the first factor descends to $E$, and commutes with the projection map $\pi$. Moreover, it preserves the linear structure on the fibers. Hence there is a representation of $G$ on the vector space of sections $\Gamma(E)$, and this is the representation of $G$ induced by $\alpha$.

We use the terminology of [KL01, Sec. 2]. (However, we replace quasiisometrically conjugate by the shorter and more accurate term quasi-conjugate.)

We will work with generalized metrics taking values in $[0,+\infty]$. A finite component of a generalized metric space is an equivalence class of points with pairwise finite distances. Clearly, quasi-isometries respect finite components.

Let $\left\{X_{i}\right\}_{i \in I}$ be a finite collection of unbounded metric spaces in the usual sense; i.e. the metric on each $X_{i}$ takes only finite values. On their product $\prod_{i \in I} X_{i}$ we consider the natural $\left(L^{2}\right.$-)product metric. On their disjoint union $\bigsqcup_{i \in I} X_{i}$ we consider the generalized metric which induces the original metric on each component $X_{i}$ and gives distance $+\infty$ to any pair of points in different components.

We observe that a quasi-isometry $\prod_{i \in I} X_{i} \rightarrow \prod_{i \in I} X_{i}^{\prime}$ preserving the product structure gives rise to a quasi-isometry $\bigsqcup_{i \in I} X_{i} \rightarrow \bigsqcup_{i \in I} X_{i}^{\prime}$, well-defined up to a bounded error, and vice versa. Thus equivalence classes of quasi-actions $\alpha: G \curvearrowright$ $\prod_{i \in I} X_{i}$ preserving the product structure correspond one-to-one to quasi-actions $\beta: G \curvearrowright \bigsqcup_{i \in I} X_{i}$. In what follows we will prove the disjoint union analog of Theorem 1.2. (The index of $H$ can be arbitrary from now on.)

Lemma 2.1. Suppose that $Y$ is a generalized metric space and that $G \curvearrowright Y$ is a quasi-action such that $G$ acts transitively on the set of finite components of $Y$. Let 
$Y_{0}$ be one of the finite components and $H$ its stabilizer in $G$. Then the restricted action $H \curvearrowright Y_{0}$ determines the action $G \curvearrowright Y$ up to quasi-conjugacy.

Proof. If $G \curvearrowright Y^{\prime}$ is another quasi-action, $Y_{0}^{\prime}$ is a finite component with stabilizer $H$, then any quasi-conjugacy between $H \curvearrowright Y_{0}$ and $H \curvearrowright Y_{0}^{\prime}$ extends in a straightforward way to a quasi-conjugacy between $G \curvearrowright Y$ and $G \curvearrowright Y^{\prime}$.

We will now show how to recover the $G$-quasi-action from the $H$-quasi-action by quasifying the construction of induced actions as described above.

Definition 2.2. An $(L, A)$-coarse fibration $(Y, \mathcal{F})$ consists of a (generalized) metric space $Y$ and a family $\mathcal{F}$ of subsets $F \subset Y$, the coarse fibers, with the following properties:

(1) The union $\bigcup_{F \in \mathcal{F}} F$ of all fibers has Hausdorff distance $\leq A$ from $Y$.

(2) For any fibers $F_{1}, F_{2} \in \mathcal{F}$ we have

$$
d_{H}\left(F_{1}, F_{2}\right) \leq L \cdot d\left(y_{1}, F_{2}\right)+A \quad \forall y_{1} \in F_{1} .
$$

We also say that $\mathcal{F}$ is a coarse fibration of $Y$.

Note that the coarse fibers are not required to be disjoint.

It follows from part (2) of the definition that $d_{H}\left(F_{1}, F_{2}\right)<+\infty$ if and only if $F_{1}$ and $F_{2}$ meet the same finite component of $Y$. We will equip the "base space" $\mathcal{F}$ with the Hausdorff metric.

Lemma 2.3. If $H \curvearrowright Y$ is an $(L, A)$-quasi-action, then the collection of quasi-orbits $O_{y}:=H \cdot y$ forms an $(L, 3 A)$-coarse fibration of $Y$.

Proof. For $h, h_{1}, h_{2} \in H$ and $y_{1}, y_{2} \in Y$ we have

$$
\begin{aligned}
d\left(h y_{1},\left(h h_{1}^{-1} h_{2}\right) y_{2}\right) & \leq d\left(\left(h h_{1}^{-1}\right)\left(h_{1} y_{1}\right),\left(h h_{1}^{-1}\right)\left(h_{2} y_{2}\right)\right)+2 A \\
& \leq L \cdot d\left(h_{1} y_{1}, h_{2} y_{2}\right)+3 A
\end{aligned}
$$

and so

$$
d\left(O_{y_{1}}, O_{y_{2}}\right) \leq L \cdot d\left(h_{1} y_{1}, O_{y_{2}}\right)+3 A .
$$

Let $(Y, \mathcal{F})$ and $\left(Y^{\prime}, \mathcal{F}^{\prime}\right)$ be coarse fibrations. We say that a map $\phi: Y \rightarrow Y^{\prime}$ quasi-respects the coarse fibrations if the image of each fiber $F \in \mathcal{F}$ is uniformly Hausdorff close to a fiber $F^{\prime} \in \mathcal{F}^{\prime}, d_{H}\left(\phi(F), F^{\prime}\right) \leq C$. The map $\phi$ then induces a map $\bar{\phi}: \mathcal{F} \rightarrow \mathcal{F}^{\prime}$ which is well-defined up to a bounded error $\leq 2 C$. Observe that if $\phi$ is an $(L, A)$-quasi-isometry, then $\bar{\phi}$ is an $(L, A+2 C)$-quasi-isometry.

We say that a quasi-action $\rho: G \curvearrowright Y$ quasi-respects a coarse fibration $\mathcal{F}$ if all maps $\rho(g)$ quasi-respect $\mathcal{F}$ with uniformly bounded error. The quasi-action $\rho$ then descends to a quasi-action $\bar{\rho}: G \curvearrowright \mathcal{F}$ which is unique up to equivalence (cf. KL01, Definition 2.3]).

We apply these general remarks to the following situation in order to obtain our main construction.

Let $G$ be a group, $H<G$ a subgroup (of arbitrary index) and $H \stackrel{\alpha}{\curvearrowright} X$ an $(L, A)$-quasi-action. Let $Y=G \times X$ where $G$ is given the metric $d\left(g_{1}, g_{2}\right)=+\infty$ unless $g_{1}=g_{2}$. That is, $Y$ consists of $|G|$ finite components each of which is a copy of $X$. The quasi-action $\alpha$ gives rise to a product quasi-action $H \stackrel{\rho_{H}}{\curvearrowright} Y$ via

$$
\rho_{H}(h,(g, x))=\left(g h^{-1}, h x\right) .
$$


We denote by $\mathcal{F}_{H}$ the coarse fibration of $Y$ by $H$-quasi-orbits. The isometric $G$ action given by

$$
\tilde{\rho}_{G}\left(g^{\prime},(g, x)\right)=\left(g^{\prime} g, x\right)
$$

commutes with $\rho_{H}$. As a consequence, $\tilde{\rho}_{G}$ descends to an isometric action

$$
\hat{\beta}:=\bar{\rho}_{G}: G \curvearrowright \mathcal{F}_{H} \text {. }
$$

If $H=G$, then $\alpha$ is quasi-conjugate to $\hat{\beta}$ via the quasi-isometry $x \mapsto \rho_{H}(H) \cdot(e, x)$. This case is used to prove Corollary 1.1, where $X^{\prime}=\mathcal{F}_{H}$.

In general, the finite components of $\mathcal{F}_{H}$ correspond to the left $H$-cosets in $G$. More precisely, $g H$ corresponds to $\bigcup_{x \in X} \rho_{H}(H) \cdot(g, x)$, that is, to the union of $\rho_{H^{-}}$ quasi-orbits contained in $g H \times X$. $H$ stabilizes the finite component $\bigcup_{x \in X} \rho_{H}(H)$. $(e, x)$. The action of $H$ on this component is quasi-conjugate to $\alpha$.

As remarked in the beginning of this section, $\hat{\beta}$ is the unique $G$-quasi-action up to quasi-conjugacy such that $G$ acts transitively on finite components and such that $H$ is the stabilizer of a finite component and the restricted $H$-quasi-action is quasi-isometrically conjugate to $\alpha$.

Passing back from disjoint unions to products we obtain Theorem 1.2

\section{Quasi-isometries AND the Classification into types (1)-(4)}

We now prove:

Lemma 3.1. Suppose $Y$ and $Y^{\prime}$ are spaces of one of the types (1)-(4) as in Theorem 1.5. If $Y$ is quasi-isometric to $Y^{\prime}$, then they have the same type, unless one is a quasi-isometrically rigid rank 1 symmetric space, and the other is of type (4).

Proof. First suppose one of the spaces is not Gromov hyperbolic. Since Gromov hyperbolicity is quasi-isometry invariant, both spaces must be higher rank spaces of either type (1) or (2). But by KL97, two irreducible symmetric spaces or Euclidean buildings of rank at least two are quasi-isometric iff they are homothetic. Thus in this case they must have the same type.

Now assume both spaces are Gromov hyperbolic. Then $\partial Y$ and $\partial Y^{\prime}$ are homeomorphic.

If $Y$ is a bounded valence bushy tree, then it is well-known that $Y$ is quasiisometric to a trivalent tree, and $\partial Y$ is homeomorphic to a Cantor set. Therefore $Y$ cannot be quasi-isometric to a space of type (1), since the boundary of a Gromov hyperbolic symmetric space is a sphere. Also, the quasi-isometry group of a trivalent tree $T$ has an induced action on the space of triples in $\partial T$ which is not proper, and hence it cannot be quasi-isometric to a space of type (4).

If $Y$ is a hyperbolic or complex hyperbolic space, then the induced action of $\mathrm{QI}(X)$ on the space of triples in $\partial X$ is not proper, and hence $Y$ cannot be quasiisometric to a space of type (4).

The lemma follows.

\section{REFERENCES}

[Ahl02] A. R. Ahlin, The large scale geometry of products of trees, Geom. Dedicata 92 (2002), 179-184, Dedicated to John Stallings on the occasion of his 65th birthday. MR 1934017 (2003j:20041)

[BKM] M. Bonk, B. Kleiner, and S. Merenkov, Rigidity of Schottky sets, Amer. J. Math., to appear. 
[BP00] M. Bourdon and H. Pajot, Rigidity of quasi-isometries for some hyperbolic buildings, Comment. Math. Helv. 75 (2000), no. 4, 701-736. MR.1789183 (2003a:30027)

[Cho96] R. Chow, Groups quasi-isometric to complex hyperbolic space, Trans. Amer. Math. Soc. 348 (1996), no. 5, 1757-1769. MR.1329530 (96h:20071)

[CJ94] A. Casson and D. Jungreis, Convergence groups and Seifert fibered 3-manifolds, Invent. Math. 118 (1994), no. 3, 441-456. MR1296353 (96f:57011)

[Gab92] D. Gabai, Convergence groups are Fuchsian groups, Ann. of Math. (2) 136 (1992), no. 3, 447-510. MR.1189862 (93m:20065)

[Gro] M. Gromov, Hyperbolic manifolds, groups and actions, Riemann surfaces and related topics: Proceedings of the 1978 Stony Brook Conference (State Univ. New York, Stony Brook, N.Y., 1978), Ann. of Math. Stud., vol. 97, Princeton Univ. Press, Princeton, NJ, 1981, pp. 183-213. MR624814 (82m:53035)

[Hin90] A. Hinkkanen, Abelian and nondiscrete convergence groups on the circle, Trans. Amer. Math. Soc. 318 (1990), no. 1, 87-121. MR1000145 (91g:30025)

[KKL98] M. Kapovich, B. Kleiner, and B. Leeb, Quasi-isometries and the de Rham decomposition, Topology 37 (1998), no. 6, 1193-1211. MR1632904 (99g:53048)

[KKLS] M. Kapovich, B. Kleiner, B. Leeb, and R. Schwartz, private communication.

[KL97] B. Kleiner and B. Leeb, Rigidity of quasi-isometries for symmetric spaces and Euclidean buildings, Inst. Hautes Études Sci. Publ. Math. (1997), no. 86, 115-197 (1998). MR 1608566 (98m:53068)

[KL01] Groups quasi-isometric to symmetric spaces, Comm. Anal. Geom. 9 (2001), no. 2, 239-260. MR.1846203 (2002d:53066)

[Lee00] B. Leeb, A characterization of irreducible symmetric spaces and Euclidean buildings of higher rank by their asymptotic geometry, Bonner Mathematische Schriften [Bonn Mathematical Publications], 326, Universität Bonn Mathematisches Institut, Bonn, 2000. MR 1934160(2004b:53060)

[Mar06] V. Markovic, Quasisymmetric groups, J. Amer. Math. Soc. 19 (2006), no. 3, 673-715 (electronic). MR2220103 (2007c:37057)

[Mos73] G. D. Mostow, Strong rigidity of locally symmetric spaces, Ann. Math. Stud., vol. 78, Princeton University Press, Princeton, NJ; University of Tokyo Press, Tokyo, 1973. MR0385004(52:5874)

[MSW03] L. Mosher, M. Sageev, and K. Whyte, Quasi-actions on trees. I. Bounded valence, Ann. of Math. (2) 158 (2003), no. 1, 115-164. MR.1998479 (2004h:20055)

[Pan89] P. Pansu, Métriques de Carnot-Carathéodory et quasiisométries des espaces symétriques de rang un, Ann. of Math. (2) 129 (1989), no. 1, 1-60. MR979599 (90e:53058)

[Sul81] D. Sullivan, On the ergodic theory at infinity of an arbitrary discrete group of hyperbolic motions, Riemann surfaces and related topics: Proceedings of the 1978 Stony Brook Conference (State Univ. New York, Stony Brook, NY, 1978), Ann. of Math. Stud., vol. 97, Princeton Univ. Press, Princeton, NJ, 1981, pp. 465-496. MR624833(83f:58052)

[Tuk86] P. Tukia, On quasiconformal groups, J. Analyse Math. 46 (1986), 318-346. MR861709 (87m:30043)

[Xie06] X. Xie, Quasi-isometric rigidity of Fuchsian buildings, Topology 45 (2006), no. 1, 101169. MR2170496 (2006m:53064)

Department of Mathematics, Yale University, New Haven, Connecticut 06520

Current address: Courant Institute of Mathematical Sciences, 251 Mercer Street, New York, New York 10012-1185

E-mail address: bkleiner@cims.nyu.edu

Mathematisches Institut, Universität München, Theresienstr. 39, D-80333 München, GERMANY

E-mail address: b.1@lmu.de 